\title{
Agenesis of Urinary Bladder with Solitary Kidney, Ectopic Ureter, and Unicornuate Uterus in an Adult Patient
}

\author{
S. M. Gowtham ${ }^{1}$ \\ M. Kiran ${ }^{1}$ \\ G. Ramkumar ${ }^{1}$ \\ D. Sunilkumar ${ }^{1}$ \\ D. Sathwik ${ }^{1}$ \\ A. Karthik ${ }^{1}$
}

\author{
${ }^{1}$ Department of Radiodiagnosis, Jawaharlal Institute of \\ Postgraduate Medical Education and Research (JIPMER), \\ Puducherry, India
}

J Gastrointestinal Abdominal Radiol ISGAR 2019;2:155-158

A 20-year-old female presented to the Urology Department at Jawaharlal Institute of Postgraduate Medical Education and Research (JIPMER) hospital with persistent urine leak since birth. She had no history of normal voiding of urine and had no recurrent urinary tract infection (UTI). Developmentally she was normal. She had abnormal gait with inability to flex right hip and knee joint. On examination, she had separate urethral and vaginal openings appearing stenosed with labial adhesions. Dribbling of urine was noted from the urethral opening and not vaginal opening.

Ultrasound of abdomen and pelvis performed with Esaote, My Laboratory 60, Genoa, Italy showed left solitary kidney without dilatation of the pelvicalyceal system or ureter in normal location with nonvisualization of urinary bladder. Contrast-enhanced computed tomography (CT) abdomen was performed on a 64 slice CT scanner (Somatom Sensation, Siemens, Erlangen, Germany). Plain imaging was performed from domes of diaphragm to proximal thigh, followed by corticomedullary phase (30-second delay), nephrographic phase (100-second delay), and excretory phase (10-minute delay) after the administration of contrast medium (iohexol $300 \mathrm{mg} / \mathrm{mL}$ iodine concentration-Omnipaque, GE Healthcare, Marlborough, MA, United States), through a $18 \mathrm{G}$ catheter secured in antecubital vein, at a dose of $1.5 \mathrm{~mL} / \mathrm{kg}$ $(80 \mathrm{~mL})$, rate of $3.5 \mathrm{~mL} / \mathrm{s}$. Saline flush was done with $20 \mathrm{~mL}$ of normal saline following contrast at same flow rate. Multiplanar reformation and volume rendering of the images were performed as needed in excretory phase. Imaging showed absent right kidney with compensatory hypertrophy of the left kidney without hydronephrosis. Urinary bladder was absent and also distal left ureter was dilated ( - Fig. 1A). The ureter was seen ectopically opening and delayed imaging in excretory phase showed contrast extravasation anterior to the vaginal wall ( - Fig. 1B). Other anomalies noted include scoliosis, dysplasia of right hip ( - Fig. 2 ), unicornuate uterus.

Magnetic resonance imaging (MRI) was performed on 1.5 Tesla scanner (Magnetom Avanto, Siemens, Erlangen, Germany). T2-weighted sagittal, axial images, T1-weighted

\section{received}

May 30, 2019

accepted after revision

September 3, 2019
Address for correspondence M. Kiran, DNB, Department of Radiodiagnosis, Jawaharlal Institute of Postgraduate Medical Education and Research (JIPMER), Puducherry, India (e-mail: kirandmk@gmail.com).

axial, short tau inversion recovery coronal of abdomen and pelvis, heavily T2 weighted MR urography coronal and maximum intensity projection to delineate the course of ureter were acquired. MRI confirmed absent urinary bladder with ectopic ureteric insertion, left solitary kidney, and unicornuate uterus ( - Fig. 3 ). Both the ovaries were normal showing follicles. Cystoscopy showed left ureter opening $1 \mathrm{~cm}$ proximal to external urethral meatus with no urinary bladder in between.

She underwent cut down vaginoplasty with adhesiolysis and laparoscopic Mainz 2 urinary pouch, which is a continent urinary pouch. She was discharged and advised to follow up after 1 month. No significant complications observed at 1-month follow-up imaging that showed functioning left kidney with ureter draining into the surgical pouch ( - Fig. 4).

\section{Discussion}

Urinary bladder agenesis or nondevelopment during fetal life is a very rare congenital abnormality. There are only 65 reported cases of this rare anomaly. ${ }^{1}$ Combination of single kidney, agenesis of urinary bladder, and ectopic ureter opening is extremely rare. Most of bladder agenesis cases are associated with other complex congenital anomalies that make survival difficult. ${ }^{2}$ Single case of bladder agenesis in adult has been reported till date in English literature. ${ }^{3}$ With an incidence of 1 in $6,00,000^{4}$ patients agenesis of urinary bladder is a rare malformation of urinary tract with only 26 live births ${ }^{1}$ reported till date as most are still born due to other associated complex congenital anomalies. Because of associated complex anomalies, most of the newborns won't survive into adulthood.

Embryologically around 5th week, cloaca is divided into urogenital sinus ventrally and rectum dorsally by urorectal septum. ${ }^{5}$ The cranial vesical part of urogenital sinus forms most of the bladder. During differentiation of cloaca caudal part of mesonephric ducts is absorbed into urinary bladder wall forming the trigone. The ureters, which initially outgrow
(C)2019 Indian Society of Gastrointestinal and Abdominal Radiology
License terms

(c) (1) $\ominus \circledast$ 

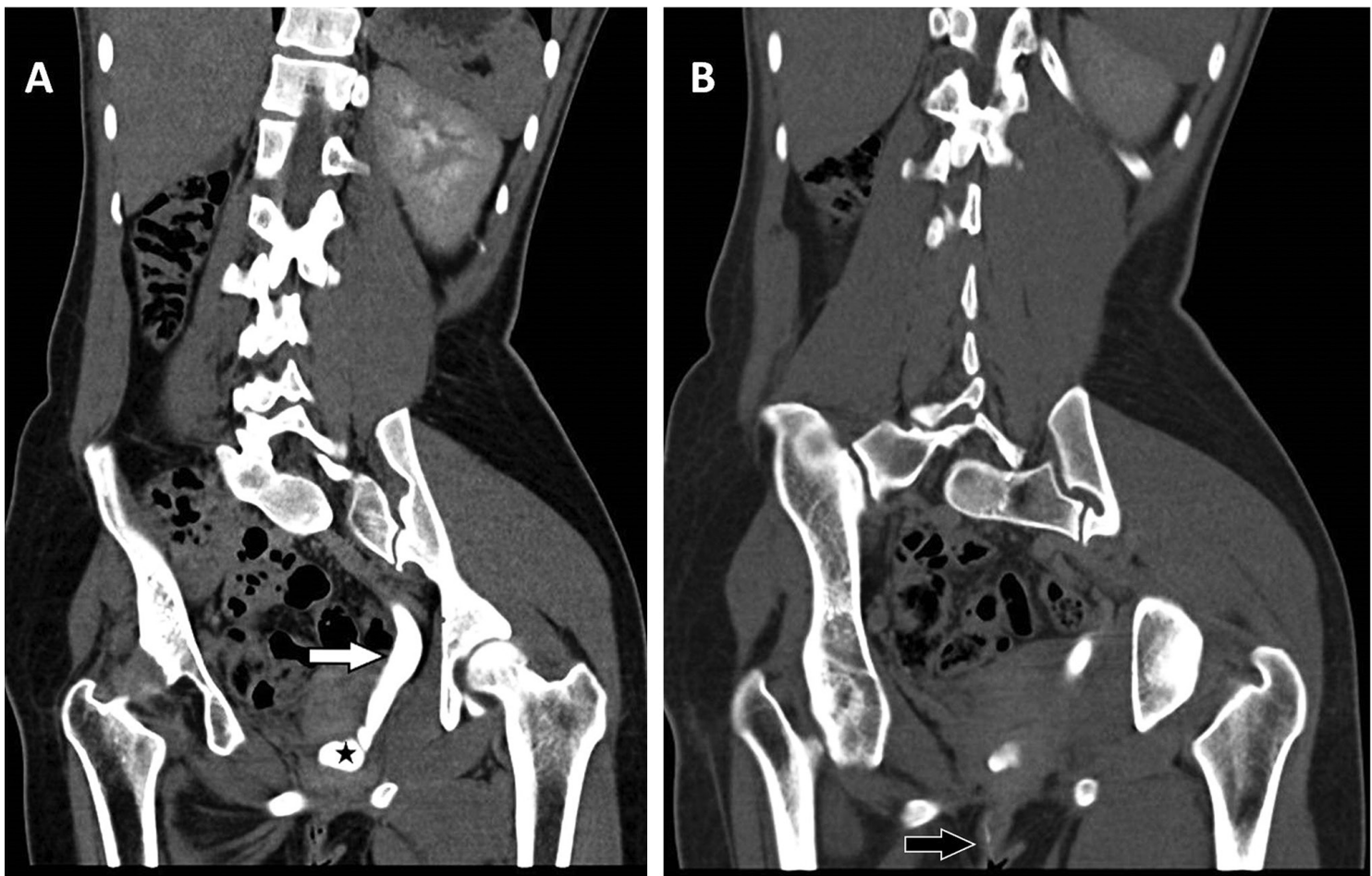

Fig. 1 Contrast-enhanced computed tomography abdomen in excretory phase showing absent urinary bladder and dilated distal left ureter (white arrow in A), ectopic location of distal end of left ureter (star in A). Thin streak of contrast is seen extravasating through the vulva (black arrow in B).

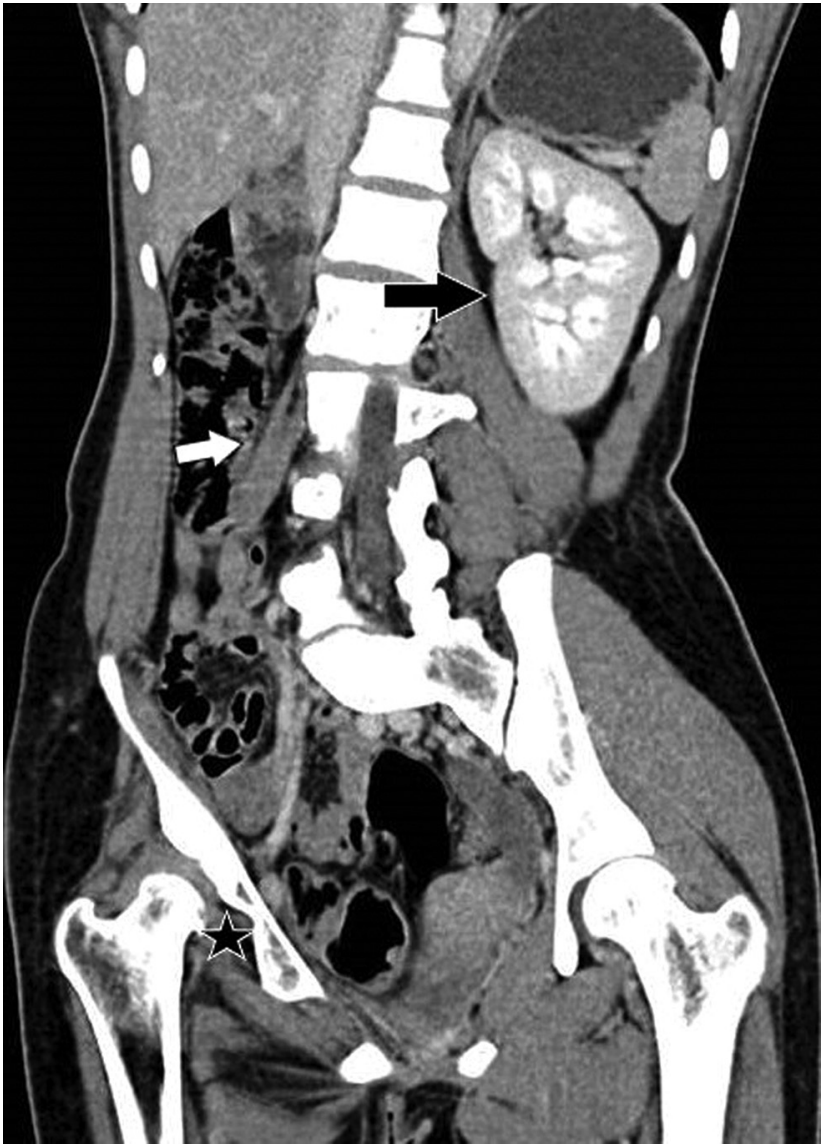

Fig. 2 Contrast-enhanced computed tomography abdomen showing dysplasia of right hip (star), solitary left kidney (black arrow), and bowel loops occupying the right renal area (white arrow).

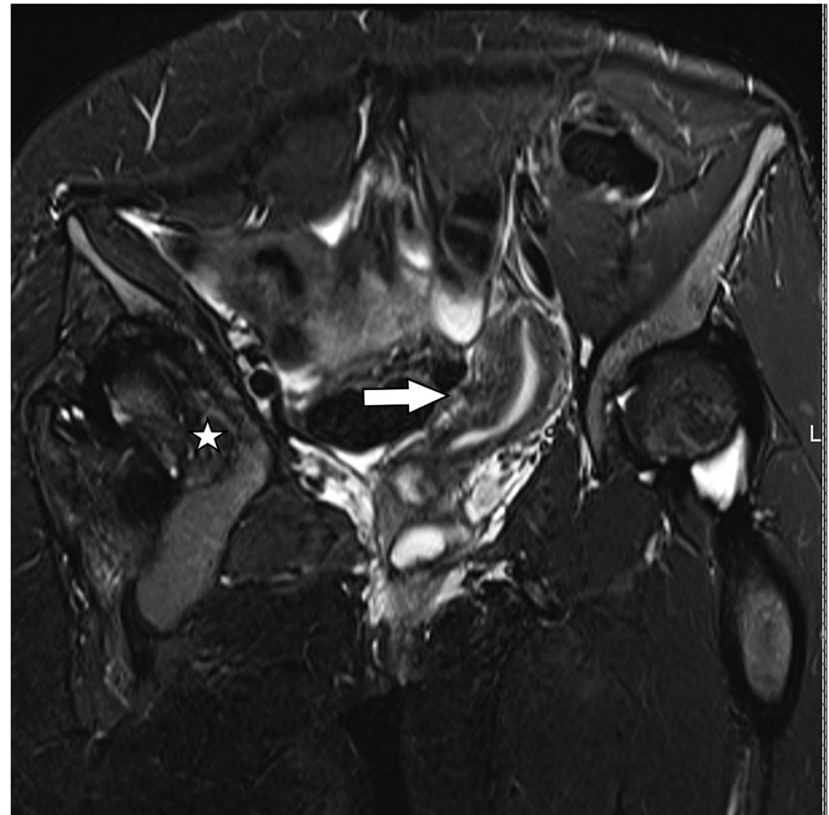

Fig. 3 Magnetic resonance imaging short tau inversion recovery coronal image showing unicornuate uterus (white arrow). Right hip dysplasia (star).

from mesonephric ducts, enter the bladder separately. Failure of incorporation of distal part of mesonephric ducts into urinary bladder may result in inadequate distension of urogenital sinus with urine resulting in atrophy of bladder.

In case of bladder agenesis in females, Mullerian structures may drain the ureters that maintain renal function due to unobstructed drainage. But in male patients with bladder 


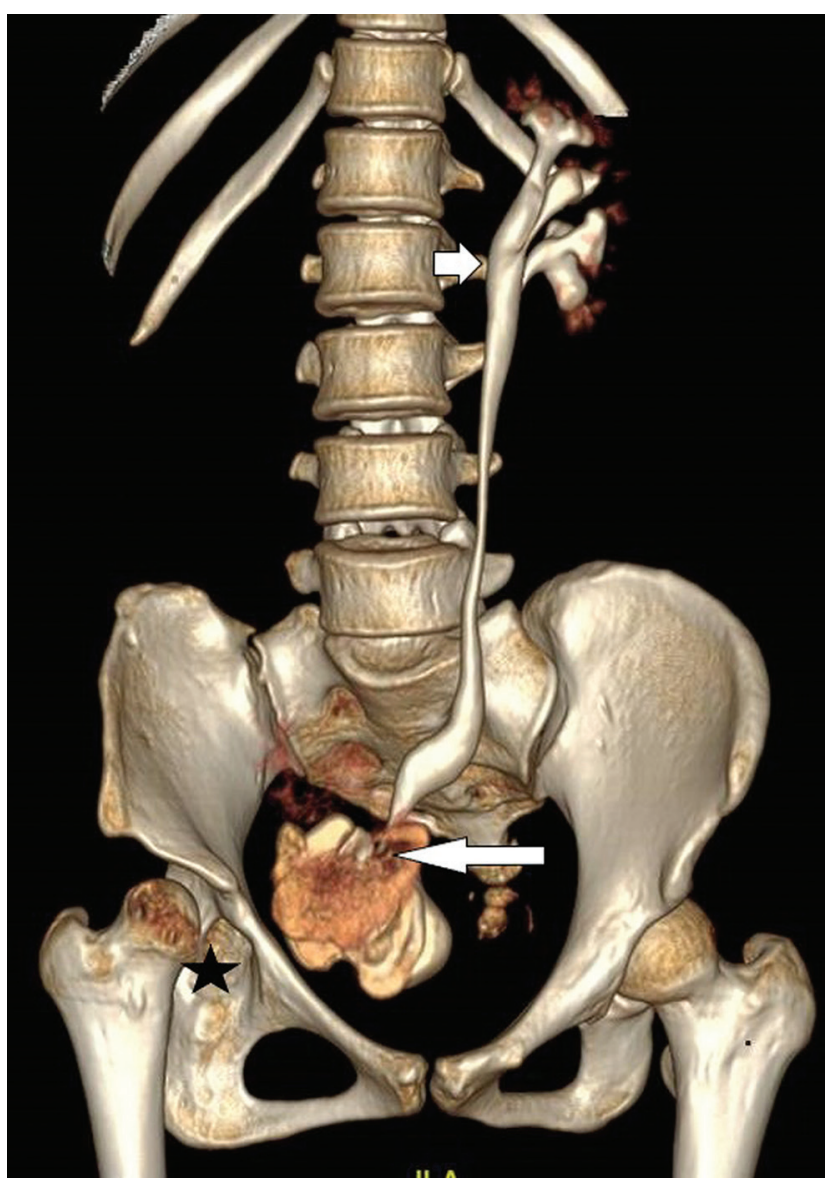

Fig. 4 Volume rendered image of follow-up computed tomography in delayed phase showing functioning left kidney (short arrow) with ureter draining into the surgical pouch (long arrow).

agenesis, options for a nonobstructed drainage include ectopic insertion into rectum or patent urachus. This explains the high female predominance in bladder agenesis.
Various associated anomalies with bladder agenesis are described in literature. Anomalies of urinary system include renal dysplasia/agenesis, morphological and positional renal anomalies, and urethral agenesis. Genital anomalies described include absent uterus, unicornuate/bicornuate uterus, and vaginal and penile agenesis. Musculoskeletal anomalies include VACTREL association, segmentation anomalies of vertebra, scoliosis, and hip dysplasia. Other anomalies described include imperforate anus, omphalocele, and variations in aorta and iliac arteries. ${ }^{1}$ Our case was associated with unicornuate uterus and anomalies of spine and musculoskeletal system.

Our patient did not have recurrent UTI and renal parameters were normal that is a rare presentation in patients of bladder agenesis with ectopic ureter insertion. She was managed with continent urinary diversion by laparoscopic procedure and no significant complications were found at 1 month follow-up.

\section{Conflict of Interest}

None declared.

\section{References}

1 Nazer II, Alhashmi G, Sharief SN, et al. A case of urinary bladder agenesis and bilateral ectopic ureters: a case report. BMC Urol 2018;18(1):83

2 Nazim SM, Zaidi Z. Bilateral ectopic ureters with bladder agenesis. J Pak Med Assoc 2012;62(10):1086-1089

3 Ghasi R, Bajaj B. Urinary bladder agenesis with bilateral single system vaginal ectopic ureters in adult. Int J Reprod Contracept Obstet Gynecol 2016;4499-4502:10.18203/2320-1770. ijrcog20164373

4 Glenn JF. Agenesis of the bladder. J Am Med Assoc 1959;169(17):2016-2018

5 Khemchandani SI. Triad of bladder agenesis with solitary kidney and ectopic ureter. Indian J Urol 2008;24(4):566-568 